\title{
NAFLD-Associated Comorbidities in Advanced Stage HCC Do Not Alter the Safety and Efficacy of Yttrium-90 Radioembolization
}

\author{
Clemens Schotten $^{a} \quad$ Lars P. Bechmann $^{\mathrm{b}}$ Paul Manka $^{\mathrm{a}} \quad$ Jens Theysohn ${ }^{\mathrm{c}}$ \\ Alexander Dechêne ${ }^{a} d \quad$ Amr El Fouly ${ }^{e}$ Francesco Barbato ${ }^{f}$ \\ Ursula Neumann $^{\text {h }}$ Sonia Radünz ${ }^{g}$ Svenja Sydor ${ }^{b}$ Dominik Heider ${ }^{h}$ \\ Marino Venerito $^{b}$ Ali Canbay ${ }^{b}$ Guido Gerken $^{a}$ Ken Herrmann ${ }^{f}$ \\ Heiner Wedemeyer ${ }^{a}$ Jan Best ${ }^{a, b}$

\begin{abstract}
aDepartment of Gastroenterology and Hepatology, Essen University Hospital, Essen, Germany; ${ }^{b}$ Department of Gastroenterology, Hepatology and Infectious Diseases, Otto von Guericke University, Magdeburg, Germany; ' Institute for Diagnostic and Interventional Radiology and Neuroradiology, Essen University Hospital, Essen, Germany; dDepartment of Internal Medicine 6, Paracelsus Medical University Nürnberg, Nürnberg, Germany; ${ }^{\mathrm{e}}$ Endemic Medicine Department, Helwan University, Cairo, Egypt; ${ }^{\mathrm{f}}$ Department of Nuclear Medicine, Essen University Hospital, Essen, Germany; ${ }^{9}$ Department of General

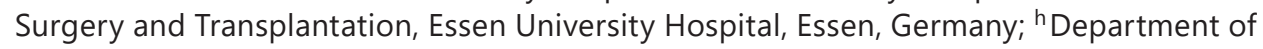
Mathematics and Computer Science, Philipps University of Marburg, Marburg, Germany
\end{abstract}

\section{Keywords}

Nonalcoholic fatty liver disease $\cdot$ Hepatitis B virus $\cdot$ Radioembolization $\cdot$ Hepatocellular carcinoma

\begin{abstract}
Background: Patients with advanced hepatocellular carcinoma (HCC) arising in nonalcoholic fatty liver disease (NAFLD) may not be suitable for systemic therapy due to metabolic syndrome-related diseases. Recent trials did not show a survival benefit of radioembolization (RE) compared to sorafenib in advanced stage HCC but RE may represent an adequate alternative in patients with contraindications to systemic therapy due to its favorable safety profile. Aim: To investigate the impact of NAFLD-related comorbidities on safety and efficacy of RE for HCC treatment in a retrospective monocentric cohort study. Patients and Methods: Safety and efficacy of RE were evaluated in patients with NAFLD-associated HCC. Hepatitis B virus (HBV)related HCC patients served as controls, exhibiting matching Barcelona Liver Cancer Clinic (BCLC) stages while showing significantly fewer metabolic comorbidities. Results: Overall, 87 HCC patients with NAFLD (mean age $71.3 \pm 6.9$ years) and 62 HCC patients with HBV (mean
\end{abstract}




\section{Liver Cancer}

\begin{tabular}{|c|c|}
\hline \multicolumn{2}{|l|}{ Liver Cancer 2019;8:491-504 } \\
\hline DOI: 10.1159/000501484 & $\begin{array}{l}\text { (c) } 2019 \text { S. Karger AG, Basel } \\
\text { www.karger.com/lic }\end{array}$ \\
\hline
\end{tabular}

Schotten et al.: Radioembolization in NAFLD-Related HCC

age $58.8 \pm 10.9$ years) not amenable to surgical or conventional locoregional treatments were included. Patients with HBV-related HCC had a comparable liver function to HCC patients with NAFLD. RE treatment-related toxicity did not differ between the two groups (increase in bilirubin Common Terminology Criteria for Adverse Events grade in 29 [38.7\%] NAFLD and 20 [39.2\%] HBV patients, $p=0.91)$. Overall survival was similar in HCC patients with NAFLD and HBV (11.1 [interquartile range, IQR, 18.27] vs. 9.3 months [IQR 14.73], $p=0.38$ ), also in the subgroup analyses of BCLC B and C stages. Conclusion: RE showed similar survival outcomes at a comparable toxicity profile in HCC patients with NAFLD and HBV. NAFLD-associated metabolic comorbidities did not exhibit limitations for RE while offering comparable therapeutic efficacy as compared to HBV patients.

(C) 2019 S. Karger AG, Basel

\section{Introduction}

Hepatocellular carcinoma (HCC) is the sixth most frequent malignancy and the second most common cause of cancer-related death worldwide [1]. Globally, HCC cases related to viral hepatitis are on the decline. In contrast, nonalcoholic fatty liver disease (NAFLD, i.e. hepatic steatosis without significant inflammation) and nonalcoholic steatohepatitis (NASH, i.e. hepatic steatosis associated with hepatic inflammation and hepatocellular ballooning) in particular have increasingly been recognized as risk factors for HCC [2]. Moreover, a significant proportion of patients develop HCC in the absence of cirrhosis [3]. Hepatocarcinogenesis appears to be associated with pathophysiological changes related to NASH [4-6]. Despite advances in screening and numerous therapeutic options, the 5-year survival rate of HCC patients is poor with approximately 15\% [7].

For patients not eligible for curative treatment approaches such as resection or liver transplantation, locoregional or systemic treatment options are indicated by the algorithm of the Barcelona Liver Cancer Clinic (BCLC) [8]. Standards of care are radiofrequency or microwave ablation for small tumors and transarterial chemoembolization (TACE) for larger tumors in the absence of macrovascular invasion. TACE prolongs survival in patients with intermediate stage HCC (BCLC stage B) [9] but has failed to show survival benefit in patients with advanced HCC, even in those individuals with well-preserved liver function (Child-Pugh A) [10].

The Current European Association for the Study of the Liver (EASL) HCC clinical practice guideline [11] recommends systemic treatment with sorafenib or lenvatinib - both tyrosine kinase inhibitors (TKI) - as first-line therapy for advanced stage HCC (BCLC C) [12]. Potential side effects related to both therapies such as hand-foot skin syndrome, diarrhea and arterial hypertension have a clinically meaningful impact on patients' quality of life with frequent necessity of dose reduction or even therapy discontinuation.

In several retrospective trials radioembolization (RE) is effective for treatment of locally advanced HCC, with fewer side effects and better quality of life compared to sorafenib [13-17].

In HCC patients with NAFLD, systemic treatment approaches are additionally limited by associated metabolic syndrome, which includes obesity, diabetes, hyperlipidemia and arterial hypertension frequently accompanied by chronic kidney and cardiovascular disease. In this scenario, standard of care treatment with TKI frequently aggravates hypertension and may increase the risk of myocardial infarction, potentially necessitating treatment de-escalation or even permanent discontinuation. Therefore, especially in this patient cohort an alternative treatment approach with a more favorable safety profile is highly desirable. 


\section{Liver Cancer}

\begin{tabular}{|c|c|}
\hline \multicolumn{2}{|l|}{ Liver Cancer 2019;8:491-504 } \\
\hline DOI: 10.1159/000501484 & $\begin{array}{l}\text { (c) } 2019 \text { S. Karger AG, Basel } \\
\text { www.karger.com/lic }\end{array}$ \\
\hline
\end{tabular}

Schotten et al.: Radioembolization in NAFLD-Related HCC

In this retrospective monocenter cohort study, we investigated the impact of NAFLDrelated comorbidities on the safety and efficacy of RE for HCC treatment. Patients with hepatitis B virus (HBV) infection-associated hepatopathy and HCC served as controls. The HBV cohort is rather homogeneous with controlled underlying disease while showing comparable liver function and BCLC tumor characteristics compared to the NAFLD patients.

\section{Materials and Methods}

454 consecutive patients with advanced HCC who underwent RE at the University Hospital Essen, Germany, between January 2006 and May 2014 were screened for this retrospective observational singlecenter study. The study was approved by the local ethics committee and carried out in accordance with the 1964 Helsinki Declaration.

In this HCC RE cohort, 87 NAFLD and $62 \mathrm{HBV}$ patients were identified and subjected to further investigation. Patients were identified by using a structured database query using the coding by German adoption of the international classification of procedures in medicine and international statistical classification of diseases and related health problems.

RE indication and suitability for yittrium-90 glass microsphere injection was verified by a multidisciplinary tumor board. Patients with hepatic tumor progression following TACE or sorafenib treatment or ineligible for surgical resection, TACE or local ablation were considered suitable for RE. Patients with evidence of portal vein obstruction or extensive tumor burden were considered eligible for RE in cases with total tumor volume not exceeding $70 \%$ of total liver volume. Prognostically relevant extrahepatic tumor burden or angiographically proven uncorrectable gastrointestinal or pulmonary shunts were considered as exclusion criteria for RE. In the absence of prognostically relevant extrahepatic tumor burden, patients who were not amenable for or refused TKI treatment, RE was offered as salvage therapy. Application of yttrium-90 microspheres was undertaken with segmental, lobar or bilobar infusions depending on tumor location and focality. Bilobar applications were predominantly administered sequentially with an interval of 4 weeks. Furthermore, patients undergoing RE in a curative intent, for bridging or downstaging to liver transplantation, were excluded.

Tumor burden and distribution according to baseline contrast-enhanced computer tomography of the thorax and abdomen or of the thorax and contrast-enhanced magnetic resonance imaging of the abdomen and a skeletal scintigraphy were recorded. Liver function and general health status were evaluated using laboratory blood tests including blood count, INR, bilirubin, ALT, AST, AP, GGT, albumin, CRP, AFP and creatinine. Glomerular filtration rate was calculated using the Modification of Diet in Renal Disease formula. Clinical parameters such as ascites, hepatic encephalopathy, body weight and size as well as preexisting medical disorders were recorded accordingly. Performance status was evaluated using the European Cooperative Oncology Group (ECOG) criteria.

Demographic data (age and sex), body mass index as well as detailed medical history of diabetes were also recorded. Diabetes mellitus, arterial hypertension, hyperlipidemia and the metabolic syndrome were defined according to the definitions of the Joint Scientific Statement for Harmonizing the Metabolic Syndrome [18]. NAFLD was defined according to histological features, when available, or cryptogenic cirrhosis in the presence of metabolic syndrome and absence of any history of significant alcohol intake and/or viral hepatitis [19]. Significant alcohol intake was defined as consumption of more than 2 standard drinks daily or more than 6 drinks daily on weekends for at least 5 years [20]. The presence of HBV and HCV was excluded by qualitative testing for hepatitis B core antibodies, hepatitis B surface antigen and anti-HCV antibodies. All patients with HBV were treated with the nucleoside analogs tenofovir or entecavir with dose adjustment according to GFR.

Liver cirrhosis was diagnosed using histology or typical imaging or clinical findings including ascites, hepatic encephalopathy, splenomegaly or laboratory results indicating impaired liver function.

Patients with 2 or more other diseases predisposing for cirrhosis and/or HCC were excluded from this study. HCC was diagnosed according to the EASL guidelines via histology or by two different contrast-enhanced imaging modalities [21, 22]. The BCLC staging system was used for determination of disease stage [8].

The clinical outcome of this cohort was recorded until December 2016. RE-related toxicity was determined by INR, bilirubin, albumin, creatinine, ALT, AP, GGT, CRP and albumin-bilirubin (ALBI) score changes to baseline 3 months after the last RE session. 
Table 1. Demographics for nonalcoholic fatty liver disease (NAFLD) and hepatitis B virus (HBV)

\begin{tabular}{|c|c|c|c|c|}
\hline Characteristics & Parameter/grade/stage & NAFLD & HBV & $p$ value \\
\hline Age & years & $71.3( \pm 6.9)$ & $58.8( \pm 10.9)$ & $\leq 0.01$ \\
\hline \multirow[t]{2}{*}{ Sex } & male/female & $74(85.1 \%) /$ & $51(82.3 \%) /$ & 0.65 \\
\hline & & $13(14.9 \%)$ & $10(17.7 \%)$ & \\
\hline \multirow[t]{4}{*}{ ECOG } & 0 & $50(57.5 \%)$ & $45(72.6 \%)$ & 0.07 \\
\hline & I & $31(35.6 \%)$ & $14(22.6 \%)$ & \\
\hline & II & $6(6.9 \%)$ & $2(3.2 \%)$ & \\
\hline & III & 0 & $1(0.7 \%)$ & \\
\hline Cirrhosis & & $44(50.6 \%)$ & $45(72.6 \%)$ & 0.01 \\
\hline \multirow[t]{2}{*}{ Child-Pugh score } & A & $75(86.2 \%)$ & $46(74.2 \%)$ & 0.07 \\
\hline & $\mathrm{B}$ & $12(13.8 \%)$ & $16(25.8 \%)$ & \\
\hline \multirow[t]{3}{*}{ ALBI score } & A & $51(58.6 \%)$ & $30(48.4 \%)$ & 0.46 \\
\hline & $\mathrm{B}$ & $34(39.1 \%)$ & $30(48.4 \%)$ & \\
\hline & $\mathrm{C}$ & $2(2.3 \%)$ & $2(3.2 \%)$ & \\
\hline \multirow[t]{3}{*}{ Ascites } & absent & $72(82.8 \%)$ & $43(69.4 \%)$ & 0.15 \\
\hline & grade I-II & $12(13.8 \%)$ & $15(24.2 \%)$ & \\
\hline & grade III-IV & $3(3.4 \%)$ & $4(6.5 \%)$ & \\
\hline Encephalopathy & history & & $1(1.6 \%)$ & 0.24 \\
\hline Esophageal varices & evidence & $21(24.1 \%)$ & $21(34.4 \%)$ & 0.17 \\
\hline Splenomegaly & evidence & $45(52.3 \%)$ & $36(60 \%)$ & 0.4 \\
\hline \multirow[t]{3}{*}{ Number of nodules } & 1 nodule & $18(20.7 \%)$ & $5(8.1 \%)$ & 0.11 \\
\hline & 2-5 nodules & $45(51.7 \%)$ & $38(61.3 \%)$ & \\
\hline & $>5$ nodules & $24(27.6 \%)$ & $19(30.6 \%)$ & \\
\hline Size of major nodule & $\mathrm{cm}$ & $9.12( \pm 11.85)$ & $6.7( \pm 4.06)$ & 0.05 \\
\hline \multirow[t]{3}{*}{ Tumor volume } & $<400 \mathrm{~mL}$ & $44(52.4 \%)$ & $33(54.1 \%)$ & 0.66 \\
\hline & $400-800 \mathrm{~mL}$ & $17(20.2 \%)$ & $15(24.6 \%)$ & \\
\hline & $>800 \mathrm{~mL}$ & $23(27.4 \%)$ & $13(21.3 \%)$ & \\
\hline Bilobar manifestation & & $54(62.1 \%)$ & $43(69.4 \%)$ & 0.36 \\
\hline Metastasis & & $13(14.9 \%)$ & $9(14.5 \%)$ & 0.94 \\
\hline Vascular infiltration & & $31(35.6 \%)$ & $27(43.5 \%)$ & 0.33 \\
\hline \multirow[t]{3}{*}{ BCLC } & $\mathrm{A}$ & $3(3.4 \%)$ & $1(1.6 \%)$ & 0.39 \\
\hline & $\mathrm{B}$ & $28(32.2 \%)$ & $26(41.9 \%)$ & \\
\hline & $\mathrm{C}$ & $56(64.4 \%)$ & $35(56.5 \%)$ & \\
\hline \multirow[t]{2}{*}{ AFP } & $<400 \mathrm{IU} / \mathrm{mL}$ & $57(65.5 \%)$ & $37(59.7 \%)$ & 0.50 \\
\hline & $>400 \mathrm{IU} / \mathrm{mL}$ & $30(34.5 \%)$ & $25(40.3 \%)$ & \\
\hline Pretreatment & & $30(34.5 \%)$ & $27(43.5 \%)$ & 0.26 \\
\hline TACE & & $16(18.4 \%)$ & $13(21 \%)$ & 0.70 \\
\hline RFA/ablation & & $5(5.7 \%)$ & $5(8.1 \%)$ & 0.60 \\
\hline Resection & & $9(10.3 \%)$ & $5(8.2 \%)$ & 0.66 \\
\hline Sorafenib & & $9(10.3 \%)$ & $7.9(16.1 \%)$ & 0.30 \\
\hline Other therapy & & $1(1.1 \%)$ & $6(9.7 \%)$ & 0.02 \\
\hline
\end{tabular}

ECOG, European Cooperative Oncology Group; ALBI, albumin-bilirubin; BCLC, Barcelona Liver Cancer Clinic; AFP, $\alpha$-fetoprotein; TACE, transarterial chemoembolization; RFA, radiofrequency ablation. $p$ values for continuous normal distributed variables by Student's $t$ test; $p$ values for nonparametrical values and for categorical variables by Mann-Whitney U test; $p$ values for nominal variables by the $\chi^{2}$ test or Fisher's exact test.

Statistical analysis was performed using the commercially available software SPSS Version 24.0 (2016). Standard descriptive statistics were used to illustrate baseline characteristics. The $p$ values for continuous, parametrical baseline variables were assessed by Student's $t$ test, nonparametrical and categorical variables by the Mann-Whitney U test and nominal categorical variables by the $\chi^{2}$ test or Fisher's exact test. Survival probabilities are displayed graphically by the Kaplan-Meier method. Univariate and multivariate Cox regression analyses were constructed to test the significance of clinical and biochemical prognostic indicators of survival with $95 \%$ confidence intervals (95\% CI) for estimated parameters. All $p$ values are twosided with a significance level of 0.05 . 


\section{Liver Cancer}

\begin{tabular}{|c|c|}
\hline \multicolumn{2}{|c|}{ Liver Cancer 2019;8:491-504 } \\
\hline DOI: $10.1159 / 000501484$ & $\begin{array}{l}\text { (c) } 2019 \text { S. Karger AG, Basel } \\
\text { www.karger.com/lic }\end{array}$ \\
\hline
\end{tabular}

Schotten et al.: Radioembolization in NAFLD-Related HCC

\section{Results}

Baseline Characteristics

Eighty-seven NAFLD patients and $62 \mathrm{HBV}$ patients were analyzed in this study. NAFLD patients were older than HBV patients (median age 71.3 [ \pm 6.9$]$ vs. 58.82 years $[ \pm 10.92], p \leq$ 0.01). Extrahepatic comorbidities such as obesity, diabetes, dyslipidemia, hypertension, cardiovascular disorders and chronic kidney diseases were more frequent in NAFLD patients $(p<0.01)$. Performance status determined by ECOG did not differ significantly between the two cohorts but trended towards a better performance status in the HBV cohort. Disease characteristics of the 149 patients at baseline are shown in Tables 1 and 3.

\section{Liver Function and Laboratory Values}

HBV patients were more likely to be cirrhotic (NAFLD 50.6\% vs. HBV 72.6\%; $p \leq 0.01$ ). Liver function determined by standard staging algorithms (Child-Pugh score; model of end-stage liver disease, MELD, albumin-bilirubin, ALBI, scores) did not significantly differ between the two groups ( $p$ ranging from 0.07 to 0.68 ). Evidence of portal hypertension encompassing ascites, splenomegaly and esophageal varices was comparable ( $p$ ranging from 0.17 to 0.4 ) (Table 1 ).

Laboratory findings showed lower median platelet count and higher levels of bilirubin in the HBV cohort compared to NAFLD within nonpathological margins. In contrast, NAFLD patients exhibited significantly higher levels of serum creatinine and consecutive lower glomerular filtration rate revealing an overall higher prevalence of chronic kidney disease than their HBV pendants (Tables 2, 3).

\section{Tumor Stages}

The majority of patients was HCC treatment naïve at the time of RE (NAFLD 65.5\% vs. HBV 56.5\%). Tumor stage according to a BCLC diagnostic and treatment algorithm showed similar proportions of BCLC A (NAFLD 3.4\% vs. HBV 1.6\%), B (NAFLD 32.2\% vs. HBV 41.9\%) and C (NAFLD $64.4 \%$ vs. HBV 56.5\%) patients ( $p=0.39$ ). NAFLD-associated HCC were more likely to be unifocal (NAFLD $20.7 \%$ vs. HBV $8.1 \%, p=0.04$ ) but with larger diameters (NAFLD $9.12 \mathrm{~cm}[ \pm 11.85]$ vs. HBV $6.7 \mathrm{~cm}[ \pm 4.06], p=0.05$ ). Target tumor volume, evidence of macrovascular infiltration and evidence of extrahepatic metastasis showed no significant differences between cohorts. A total of 204 RE treatments were performed with a median of 1 session (range, 1-2) per patient. 94 RE were performed as single sessions (17 patients single session bilobar, 77 patients single session unilobar), and 55 were administered sequentially bilobar sessions. Decision to treat uni- versus bilobar and sequential versus single session was made independently of underlying hepatopathy.

\section{Safety and Tolerability}

Follow-up examinations were commonly performed by outpatient practices or at our gastroenterology outpatient clinic. Thus, laboratory examinations were not consistently available for all patients. Between 40 and $85 \%$ of patients provided evaluable laboratory results 3 months after RE. Posttreatment toxicity determined by change of Common Terminology Criteria for Adverse Events (CTCAE) or ALBI score did not differ between the two groups. Grade 3 CTCAE total bilirubin increase was reported in $20.0 \%$ of NAFLD versus $15.7 \%$ of HBV patients, respectively ( $p=0.64$ ). None of the patients experienced albumin toxicities $\geq$ grade 3 after RE (Table 2 ).

Overall Survival

NAFLD and HBV patients exhibited no significant differences in overall survival (OS) after RE analyzed by the Kaplan-Meier method (11.1 [interquartile range, IQR, 18.3] vs. 9.3 


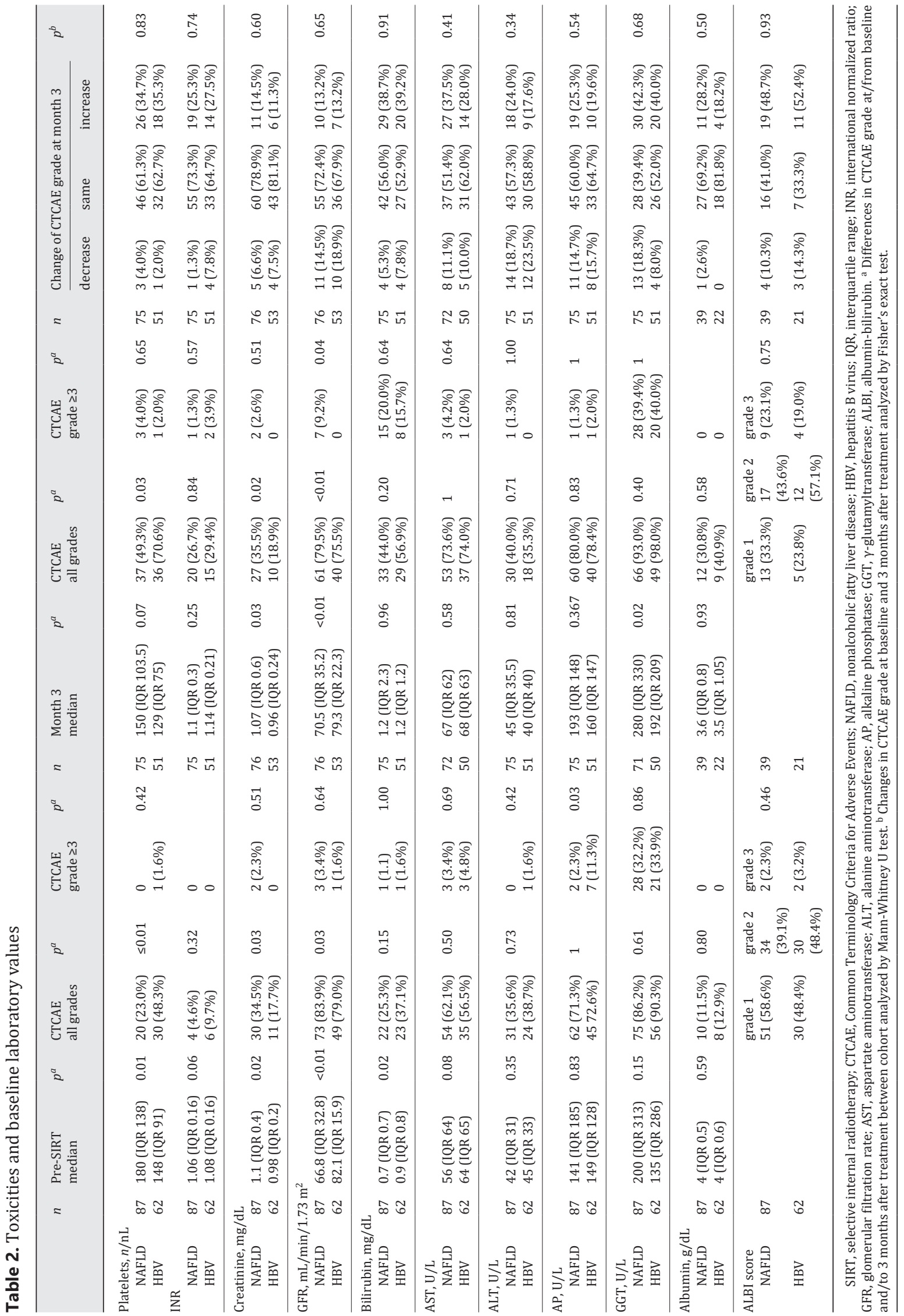


Schotten et al.: Radioembolization in NAFLD-Related HCC

Table 3. Features of metabolic syndrome and extrahepatic comorbidities

\begin{tabular}{|c|c|c|c|c|c|c|}
\hline Characteristic & NAFLD & HBV & $p$ value & & HR & $\begin{array}{l}p \\
\text { value }\end{array}$ \\
\hline \multirow[t]{2}{*}{ BMI } & $29.8( \pm 5)$ & $25.97( \pm 5.5)$ & $\leq 0.01$ & NAFLD & 0.99 (CI 0.94-1.04) & 0.68 \\
\hline & & & & HBV & 0.97 (CI 0.91-1.04) & 0.38 \\
\hline \multirow[t]{2}{*}{ Diabetes } & $54(62.1 \%)$ & $11(17.1 \%)$ & $\leq 0.01$ & NAFLD & 1.47 (CI 0.93-2.30) & 0.1 \\
\hline & & & & HBV & 0.85 (CI $0.43-1.69$ ) & 0.64 \\
\hline \multirow[t]{2}{*}{ Dyslipidemia } & $51(59.3 \%)$ & $20(32.3 \%)$ & $\leq 0.01$ & NAFLD & 0.93 (CI $0.6-1.46)$ & 0.76 \\
\hline & & & & HBV & 1.06 (CI 0.6-1.86) & 0.84 \\
\hline \multirow[t]{2}{*}{ Arterial hypertension } & $74(85.1 \%)$ & $27(43.5 \%)$ & $\leq 0.01$ & NAFLD & 0.65 (CI $0.36-1.18$ ) & 0.16 \\
\hline & & & & HBV & 1.24 (CI $0.72-2.16)$ & 0.44 \\
\hline \multirow[t]{2}{*}{ Cardiovascular disease } & $50(57.5 \%)$ & $14(22.6 \%)$ & $\leq 0.01$ & NAFLD & 1.23 (CI 0.79-1.92) & 0.35 \\
\hline & & & & HBV & 1.11 (CI $0.58-2.11$ ) & 0.75 \\
\hline \multirow[t]{2}{*}{ Chronic kidney disease } & $30(34.9 \%)$ & $8(12.9 \%)$ & $\leq 0.01$ & NAFLD & 0.8 (CI $0.50-1.27)$ & 0.35 \\
\hline & & & & HBV & 1.78 (CI 0.83-3.80) & 0.14 \\
\hline
\end{tabular}

NAFLD, nonalcoholic fatty liver disease; HBV, hepatitis B virus; HR, hazard ratio; BMI, body mass index; $\mathrm{CI}$, confidence interval. Univariate Cox proportional hazards model in features of metabolic syndrome.

Fig. 1. Kaplan-Meier graph of OS NAFLD versus HBV after RE. $p$ value by Mann-Whitney U test.

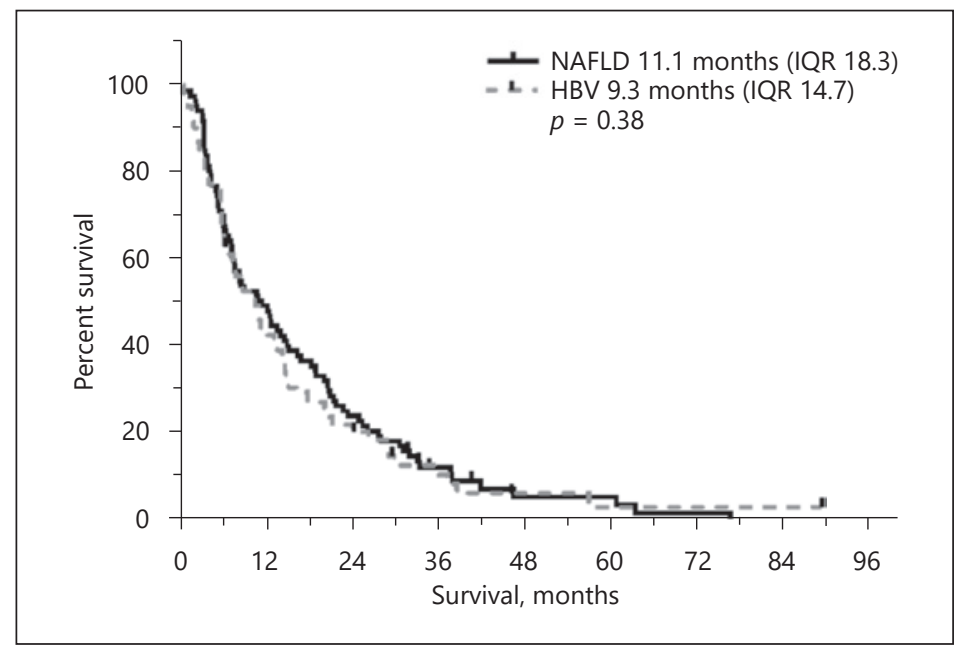

months [IQR 14.7], $p=0.38$ ) (Fig. 1). Patients at BCLC stage A were not evaluable due to the small sample size. The median OS in stage BCLC B exhibited a median survival of 16.4 months (IQR $21.5 ; n=28$ ) in NAFLD versus 14.3 months (IQR $17.6 ; n=26$ ) in HBV patients, in advanced stage BCLC C 8.0 months (IQR 16.2; $n=56$ ) in the NAFLD versus 5.6 months (IQR $8.5 ; n=35$ ) in the HBV cohort. OS was within the NAFLD $(p=0.13)$ and the HBV $(p=0.75)$ cohort independent of uni- versus bilobar treatment approach.

\section{Univariate/Multivariate Analyses}

In univariate analysis bilirubin, AST, albumin, ECOG, ascites and metastases were predictors of survival for both cohorts. Creatinine (NAFLD $p=0.09$ vs. HBV $p<0.01$ ) and vascular infiltration (NAFLD $p=0.5$ vs. HBV $p<0.01$ ) were not prognostically significant in NAFLD in contrast to HBV (Table 4). Extrahepatic comorbidities including obesity, diabetes, dyslipidemia, arterial hypertension, cardiovascular disorders and chronic kidney diseases were not predictive for OS (Fig. 2; Table 3). In NAFLD, age-adjusted subgroup analysis showed 


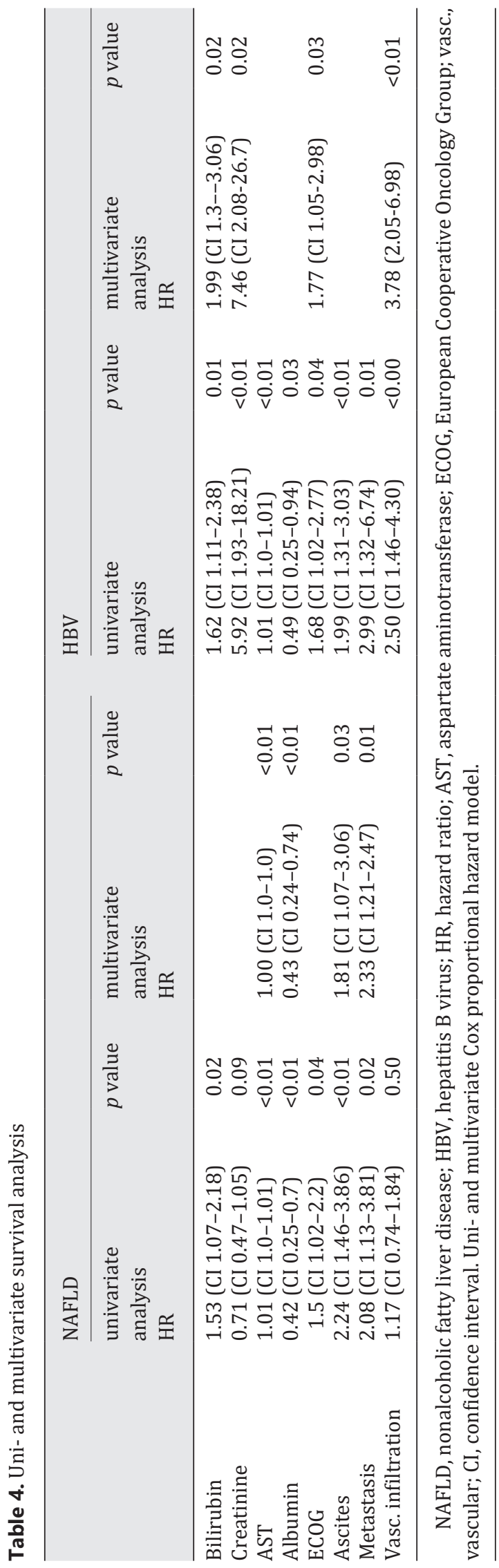




\section{Liver \\ Cancer}

Fig. 2. Kaplan-Meier graph of OS diabetes in NAFLD after RE. $p$ value and hazard ratio were calculated by the Cox proportional hazards model.

Fig. 3. Kaplan-Meier graph of creatinine dependent OS in HBV after RE. For graphic presentability creatinine was transferred to a nominal variable analog CTCAE (normal values, $\geq$ CTCAE I). Displayed $p$ value and HR were calculated by the Cox regression model for the transferred variable.

\begin{tabular}{l|l}
\hline \multicolumn{2}{l}{ Liver Cancer 2019;8:491-504 } \\
\hline DOI: 10.1159/000501484 & $\begin{array}{l}\text { @ 2019 S. Karger AG, Basel } \\
\text { www.karger.com/lic }\end{array}$ \\
\hline
\end{tabular}

Schotten et al.: Radioembolization in NAFLD-Related HCC
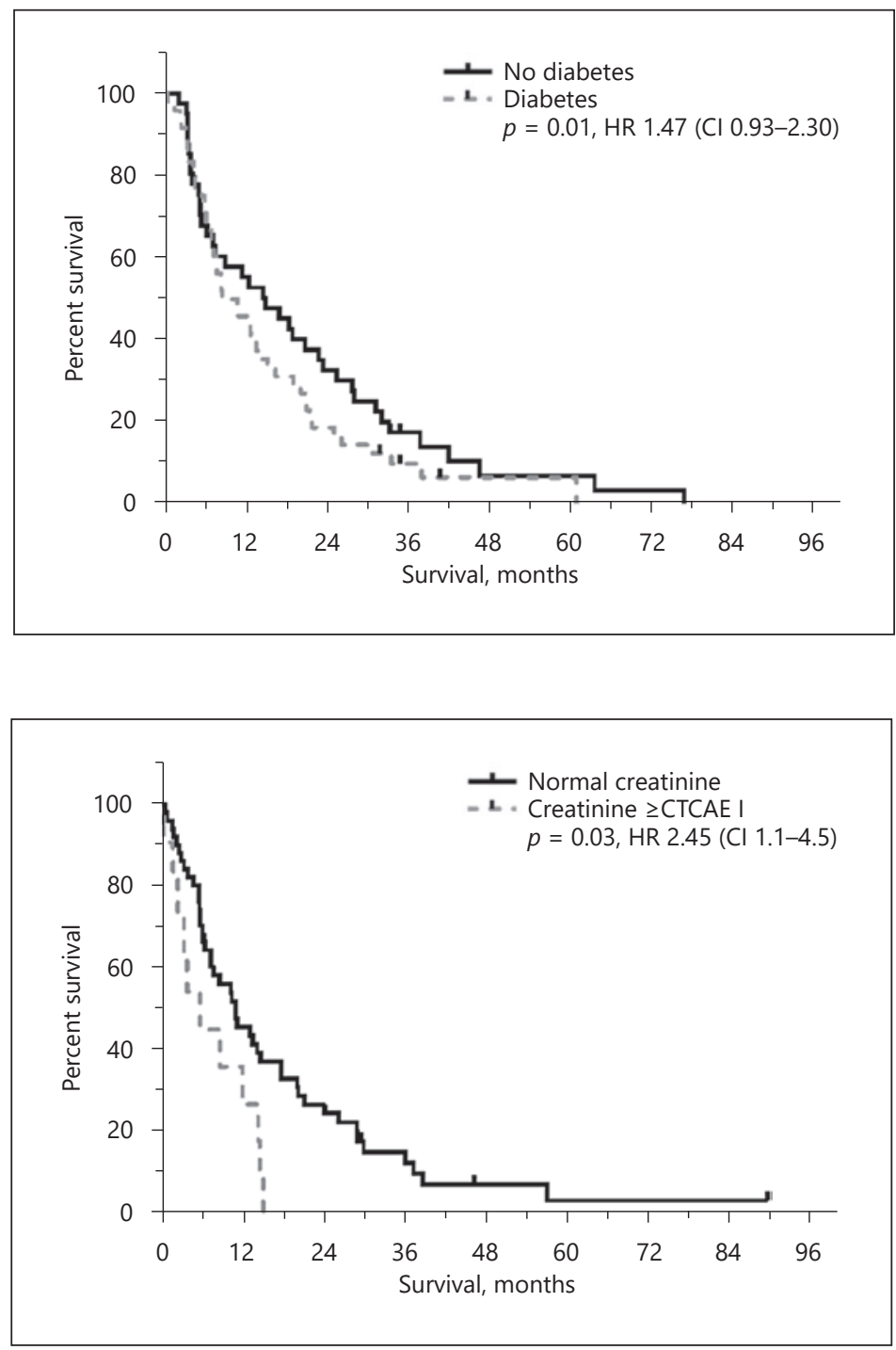

no significant differences (age <70 years OS 10.6 months vs. $>70$ years 12.1 months; $p=0.31$; hazard ratio, HR, 0.79 [CI 0.51-1.24]).

Multivariate analysis demonstrated that AST, albumin, ascites and evidence of metastases were independent negative predictors of survival in NAFLD. Bilirubin, creatine, ECOG and vascular infiltration were negative predictors in the HBV cohort, respectively (Fig. 3; Table 4).

\section{Discussion}

In our study survival outcomes and toxicity profile for HCC patients with NAFLD and HBV who received RE were comparable. While TKI treatment is frequently restricted by doselimiting toxicities, RE may represent an adequate therapeutic alternative, especially for those patients with NAFLD-related HCC. Our patients exhibited survival rates comparable to sorafenib as shown in previous phase III and IV trials [12, 23]. 


\section{Liver

Our data clearly demonstrate that NAFLD-associated comorbidities did not impair RE safety compared to our HBV controls. While renal impairment significantly reduces OS in HBV, it did not impact OS in NAFLD.

In our HCC cohort NAFLD exhibited a statistically noninferior OS compared to HBV. The numerically longer OS in NAFLD compared to HBV patients is remarkable considering the distinct higher average age in the NAFLD population.

In a recent bicentric retrospective study on older HCC patients ( $\geq 75$ years) conducted by Williet et al. [24], sorafenib treatment was discontinued in $60.8 \%$ of the patients within 3 months due to intolerable toxicity, mostly in those who had ECOG performance status $\geq \mathrm{I}$, or cardiovascular disease. In this setting, OS was significantly reduced [24]. Here, RE exhibited similar efficacy with a favorable safety profile compared to sorafenib in intermediate and advanced stage HCC independent of underlying liver disease [17, 25-28], which is in accordance with our findings.

The median OS in our RE NAFLD and HBV cohort was comparable to major phase III firstline systemic treatment trials (sorafenib monotherapy ranging from 8.0 to 12.3 months [12], lenvatinib monotherapy 13.6 months [29]) and phase III RE trials (SARAH trial with RE monotherapy 8.8 months [28], SIRVENIB trial with RE plus sorafenib 9.9 months [30]). In contrast to the systemic and locoregional phase III trials mentioned above, a larger proportion of our RE-treated patients had impaired liver function at the time of treatment classified as Child-Pugh stage B (NAFLD 13.8\% and HBV 25.8\%) and poorer ECOG performance status $>$ I (6\%). In direct comparison to our study, patients with impaired liver function were rather underrepresented in relevant phase III HCC treatment trials (Child-Pugh stage B ranging from 1 to $5 \%$ in TKI trials $[12,29]$ and from 7.7 to $16 \%$ in RE trials $[13,28])$. The majority of our NAFLD and HBV patients had advanced stage HCC (BCLC C) with limited extrahepatic tumor burden at the time of RE. According to the literature, in these patients, morbidity is mainly driven by intrahepatic tumor burden resulting in hepatic decompensation, therefore intrahepatic tumor control is a relevant positive predictive factor [31, 32].

Recent clinical trials on RE commonly include HCC patients of mixed etiologies. Among those, $\mathrm{HCV}$ patients represent the largest cohort with a proportion ranging between 43.1 and $46.1 \%[16,25,33]$. Despite increasing prevalence of NAFLD as underlying etiology of HCC in the western world, a focused analysis on safety and efficacy of RE in this cohort has not been performed yet.

According to our data NAFLD and HBV patients exhibit distinct differences in extrahepatic comorbidities. The detected manifestations of metabolic syndrome, cardiovascular disease and consecutive chronic kidney disease (CKD) in our NAFLD cohort are in accordance with the findings of previous publications [34].

Jun et al. [35] described that compared to hepatitis-virus-related HCC, the cohort with cryptogenic HCC (containing a relevant proportion of NAFLD) had larger tumor sizes and poorer OS despite lower prevalence of cirrhosis. This observation is confirmed by a retrospective German monocenter study with 1,119 HCC patients, conducted by Weinmann et al. [36], where larger and more likely unifocal tumor lesions were found in NASH compared to HBV. Furthermore, they demonstrated that the NASH cohort showed an inferior OS compared to other HCC etiologies despite preserved liver function [36]. Our cohort showed similar results to those of Weinmann et al. and Jun et. al concerning evidence of liver cirrhosis and advanced tumor stage. Of note, two thirds of our NAFLD patients were naïve to tumor specific therapy prior to RE. While in the cohort of Weinmann et al. [36] the MELD score in NASH was significantly lower compared to controls, in our study it was consistent between NAFLD and HBV. This is attributed to elevated baseline serum creatinine levels in NAFLD, resulting in a higher MELD score despite adequate liver function. In our cohort HBV and NAFLD patients exhibited matching BCLC stages. Furthermore, HBV showed favorable preconditions with 


\section{Liver
Cancer}

\begin{tabular}{|c|c|}
\hline \multicolumn{2}{|l|}{ Liver Cancer 2019;8:491-504 } \\
\hline DOI: 10.1159/000501484 & $\begin{array}{l}\text { (c) } 2019 \text { S. Karger AG, Basel } \\
\text { www.karger.com/lic }\end{array}$ \\
\hline
\end{tabular}

Schotten et al.: Radioembolization in NAFLD-Related HCC

younger age, less comorbidities and a tendency to a better ECOG performance status. Despite disadvantageous risk factors, our NAFLD patients undergoing RE showed a safety profile comparable to HBV. Hepatic toxicity expressed by ALBI score did not favor one etiology either. Also, unanticipated, median OS following RE between our NAFLD and HBV cohorts did not exhibit significant differences (NAFLD OS 11.17 months [IQR 18.27] vs. HBV OS 9.32 months [IQR 14.73]).

In accordance with the results of a multicenter study conducted by Golfieri et al. [25], where OS after RE was not affected by patient age (197 patients $<70$ years vs. 128 patients $>70$ years), in our NAFLD cohort age-adjusted subgroup analyses confirmed that patient age and related comorbidities did not impact OS. In contrast, higher age in TKI treatment is a predictor for toxicity and unfavorable survival [24]. Our data clearly indicate that NAFLD and age-related comorbidities do neither result in higher toxicity following RE nor significantly impact median OS.

Evidence on the potential impact of CKD on OS in HCC patients is limited; however, the current literature indicates that CKD is associated with poor prognosis [37]. Safety data on renal toxicity of sorafenib in HCC treatment are scarce and have to be extrapolated from other tumor entities. Available pharmacokinetic analyses from sorafenib in renal cell carcinoma treatment implicate requirement to closely monitor drug-related toxicity [38].

Most likely related to CKD and independent of liver function, NAFLD patients exhibit higher baseline serum creatinine levels, resulting in higher MELD scores, showing no correlation with OS in contrast to the HBV cohort. Here elevated creatinine (HR 7.46 [CI 2.0826.7]) with an increased MELD score substantially impacts survival since renal impairment is an expression of the multifactorial interplay in the presence of liver cirrhosis $[39,40]$. This observation emphasizes the etiology dependent heterogeneity of HCC and the dynamic interaction between hepatopathy and extrahepatic comorbidity.

To our knowledge this is the first European study selectively investigating safety and efficacy of RE in an HCC-NAFLD cohort with an adequate population size, despite its monocentric design. Data on metabolic and cardiovascular features and toxicity of NAFLD were well recorded and enabled us to clearly demonstrate that RE in NAFLD exhibited comparable survival outcomes to an HBV population, despite multimorbidity.

However, our study shows certain limitations related to the retrospective nature of this monocenter RE cohort. While several prospective RE trials systematically recorded features of quality of life confirming the favorable tolerability of RE treatment, our study focused on the assessment of parameters reflecting safety and efficacy. Furthermore, follow-up data regarding the cause of death are unavailable in our study.

\section{Conclusion}

Increasing incidence of NAFLD-related HCC demands treatment approaches accounting for comorbidities that are related to metabolic syndrome and higher patient age. RE may represent a safe and efficient treatment option especially for those who exhibit potential contraindications for standard of care locoregional and systemic treatment options.

\section{Acknowledgment}

We thank Uta Hinderberger, Jennifer Weinreich and Insa Meßerschmidt for their excellent support in data acquisition. 
Schotten et al.: Radioembolization in NAFLD-Related HCC

\section{Statement of Ethics}

The study was approved by the local ethics committee and carried out in accordance with the 1964 Helsinki Declaration. Patients were identified by numbers.

\section{Disclosure Statement}

Dr. Best, Dr. El Fouly, Prof. Dr. Dechene and Prof. Theysohn received speaker's fees from BTG (speakers bureau, consulting). Prof. Dr. Herrmann received a speaker's fee from BTG ( $1 \times$ speakers bureau), personal fees from Endocyte, Ipsen, Adacap, GE Healthcare, personal fees and nonfinancial support from Siemens Healthineers, Bayer, Curium, nonfinancial support from ABX Sofie. Prof. Dr. Wedemeyer received personal fees from Abbvie, Abbott, Janssen, Eiger, MyrGmbH, Falk, Norgine, MSD personal fees and financial support by Abbott, BMS (consulting, research support, speakers bureau), Gilead, Roche Diagnostic.

\section{Funding Sources}

This work has been supported by the Jackstaedt Foundation to Lars Bechmann and Jan Best as well as the Wilhelm Laupitz Foundation to Ali Canbay.

\section{Author Contributions}

Acquisition of data: Clemens Schotten, Jan Best, Francesco Barbato, Sonia Radünz, Jens Theysohn. Analysis and interpretation of data: Clemens Schotten, Jan Best, Ali Canbay, Francesco Barbato, Jens Theysohn, Alexander Dechêne, Marino Venerito, Amr El Fouly, Guido Gerken.

Drafting of the manuscript: Jan Best, Marino Venerito, Clemens Schotten, Alexander Dechêne, Jens Theysohn, Lars Bechmann, Heiner Wedemeyer.

Statistical analysis: Clemens Schotten, Dominik Heider, Ursula Neumann, Paul Manka.

Obtained funding: Lars Bechmann.

Technical or material support: Svenja Sydor.

Study supervision: Jan Best, Ali Canbay, Heiner Wedemeyer.

Critical revision of the manuscript for important intellectual content: all authors.

\section{References}

1 El-Serag HB. Hepatocellular carcinoma. N Engl J Med. 2011 Sep;365(12):1118-27.

2 White DL, Kanwal F, El-Serag HB. Association between nonalcoholic fatty liver disease and risk for hepatocellular cancer, based on systematic review. Clin Gastroenterol Hepatol 2012;10:1342-59. e1342.

3 Ertle J, Dechêne A, Sowa JP, Penndorf V, Herzer K, Kaiser G, et al. Non-alcoholic fatty liver disease progresses to hepatocellular carcinoma in the absence of apparent cirrhosis. Int J Cancer. 2011 May;128(10):2436-43.

4 Bechmann LP, Hannivoort RA, Gerken G, Hotamisligil GS, Trauner M, Canbay A. The interaction of hepatic lipid and glucose metabolism in liver diseases. J Hepatol. 2012 Apr;56(4):952-64.

5 El-Serag HB, Richardson PA, Everhart JE. The role of diabetes in hepatocellular carcinoma: a case-control study among United States Veterans. Am J Gastroenterol. 2001 Aug;96(8):2462-7.

6 Marrero JA, Fontana RJ, Fu S, Conjeevaram HS, Su GL, Lok AS. Alcohol, tobacco and obesity are synergistic risk factors for hepatocellular carcinoma. J Hepatol. 2005 Feb;42(2):218-24.

7 Jemal A, Bray F, Center MM, Ferlay J, Ward E, Forman D. Global cancer statistics. CA Cancer J Clin. 2011 Mar-Apr;61(2):69-90.

8 Bruix J, Sherman M; American Association for the Study of Liver Diseases. Management of hepatocellular carcinoma: an update. Hepatology. 2011 Mar;53(3):1020-2.

9 Llovet JM, Fuster J, Bruix J. Prognosis of hepatocellular carcinoma. Hepatogastroenterology. 2002 Jan-Feb; 49(43):7-11.

10 Groupe d'Etude et de Traitement du Carcinome Hépatocellulaire. A comparison of lipiodol chemoembolization and conservative treatment for unresectable hepatocellular carcinoma. N Engl J Med. 1995 May; 332(19):1256-61. 
Schotten et al.: Radioembolization in NAFLD-Related HCC

11 Galle PR, Forner A, Llovet JM, Mazzaferro V, Piscaglia F, Raoul JL, et al.; European Association for the Study of the Liver. EASL clinical practice guidelines: management of hepatocellular carcinoma. J Hepatol. 2018;69(1): 182-236.

12 Llovet JM, Ricci S, Mazzaferro V, Hilgard P, Gane E, Blanc JF, et al.; SHARP Investigators Study Group. Sorafenib in advanced hepatocellular carcinoma. N Engl J Med. 2008 Jul;359(4):378-90.

13 Cho YY, Lee M, Kim HC, Chung JW, Kim YH, Gwak GY, et al. Radioembolization Is a Safe and Effective Treatment for Hepatocellular Carcinoma with Portal Vein Thrombosis: A Propensity Score Analysis. PLoS One. 2016 May; 11(5):e0154986.

14 de la Torre MA, Buades-Mateu J, de la Rosa PA, Lué A, Bustamante FJ, Serrano MT, et al. A comparison of survival in patients with hepatocellular carcinoma and portal vein invasion treated by radioembolization or sorafenib. Liver Int. 2016 Aug;36(8):1206-12.

15 Gramenzi A, Golfieri R, Mosconi C, Cappelli A, Granito A, Cucchetti A, et al.; BLOG (Bologna Liver Oncology Group). Yttrium-90 radioembolization vs sorafenib for intermediate-locally advanced hepatocellular carcinoma: a cohort study with propensity score analysis. Liver Int. 2015 Mar;35(3):1036-47.

16 Salem R, Gabr A, Riaz A, Mora R, Ali R, Abecassis M, et al. Institutional decision to adopt Y90 as primary treatment for hepatocellular carcinoma informed by a 1,000-patient 15-year experience. Hepatology. 2018 Oct;68(4):1429-40.

17 Hilgard P, Hamami M, Fouly AE, Scherag A, Müller S, Ertle J, et al. Radioembolization with yttrium-90 glass microspheres in hepatocellular carcinoma: european experience on safety and long-term survival. Hepatology. 2010 Nov;52(5):1741-9.

18 Alberti KG, Eckel RH, Grundy SM, Zimmet PZ, Cleeman JI, Donato KA, et al.; International Diabetes Federation Task Force on Epidemiology and Prevention; Hational Heart, Lung, and Blood Institute; American Heart Association; World Heart Federation; International Atherosclerosis Society; International Association for the Study of Obesity. Harmonizing the metabolic syndrome: a joint interim statement of the International Diabetes Federation Task Force on Epidemiology and Prevention; National Heart, Lung, and Blood Institute; American Heart Association; World Heart Federation; International Atherosclerosis Society; and International Association for the Study of Obesity. Circulation. 2009 Oct;120(16):1640-5.

19 Ascha MS, Hanouneh IA, Lopez R, Tamimi TA, Feldstein AF, Zein NN. The incidence and risk factors of hepatocellular carcinoma in patients with nonalcoholic steatohepatitis. Hepatology. 2010 Jun;51(6):1972-8.

20 Dawson DA, Grant BF, Harford TC. Variation in the association of alcohol consumption with five DSM-IV alcohol problem domains. Alcohol Clin Exp Res. 1995 Feb;19(1):66-74.

21 Bruix J, Sherman M; Practice Guidelines Committee, American Association for the Study of Liver Diseases. Management of hepatocellular carcinoma. Hepatology. 2005 Nov;42(5):1208-36.

22 European Association For The Study Of The L. European Organisation For R, Treatment Of C: EASL-EORTC clinical practice guidelines: management of hepatocellular carcinoma. J Hepatol. 2012;56(4):908-43.

23 Ganten TM, Stauber RE, Schott E, Malfertheiner P, Buder R, Galle PR, et al. Sorafenib in Patients with Hepatocellular Carcinoma-Results of the Observational INSIGHT Study. Clin Cancer Res. 2017 Oct;23(19):5720-8.

24 Williet N, Clavel L, Bourmaud A, Verot C, Bouarioua N, Roblin X, et al. Tolerance and outcomes of sorafenib in elderly patients treated for advanced hepatocellular carcinoma. Dig Liver Dis. 2017 Sep;49(9):1043-9.

25 Golfieri R, Bilbao JI, Carpanese L, Cianni R, Gasparini D, Ezziddin S, et al.; European Network on Radioembolization with Yttrium-90 Microspheres (ENRY) study collaborators. Comparison of the survival and tolerability of radioembolization in elderly vs. younger patients with unresectable hepatocellular carcinoma. J Hepatol. 2013 Oct;59(4):753-61.

26 Mazzaferro V, Sposito C, Bhoori S, Romito R, Chiesa C, Morosi C, et al. Yttrium-90 radioembolization for intermediate-advanced hepatocellular carcinoma: a phase 2 study. Hepatology. 2013 May;57(5):1826-37.

27 Wang H, Wang H, Yu Z, Liu H. Alternative treatment strategies to sorafenib in patients with advanced hepatocellular carcinoma: a meta-analysis of randomized phase III trials. OncoTargets Ther. 2018 Aug;11:5195-201.

28 Vilgrain V, Pereira H, Assenat E, Guiu B, Ilonca AD, Pageaux GP, et al.; SARAH Trial Group. Efficacy and safety of selective internal radiotherapy with yttrium-90 resin microspheres compared with sorafenib in locally advanced and inoperable hepatocellular carcinoma (SARAH): an open-label randomised controlled phase 3 trial. Lancet Oncol. 2017 Dec;18(12):1624-36.

29 Kudo M, Finn RS, Qin S, Han KH, Ikeda K, Piscaglia F, et al. Lenvatinib versus sorafenib in first-line treatment of patients with unresectable hepatocellular carcinoma: a randomised phase 3 non-inferiority trial. Lancet. 2018 Mar;391(10126):1163-73.

30 Chow PK, Gandhi M, Tan SB, Khin MW, Khasbazar A, Ong J, et al.; Asia-Pacific Hepatocellular Carcinoma Trials Group. SIRveNIB: Selective Internal Radiation Therapy Versus Sorafenib in Asia-Pacific Patients With Hepatocellular Carcinoma. J Clin Oncol. 2018 Jul;36(19):1913-21.

31 Llovet JM, Bustamante J, Castells A, Vilana R, Ayuso MC, Sala M, et al. Natural history of untreated nonsurgical hepatocellular carcinoma: rationale for the design and evaluation of therapeutic trials. Hepatology. 1999 Jan; 29(1):62-7.

32 Cabibbo G, Maida M, Genco C, Parisi P, Peralta M, Antonucci M, et al. Natural history of untreatable hepatocellular carcinoma: A retrospective cohort study. World J Hepatol. 2012 Sep;4(9):256-61.

33 Spreafico C, Sposito C, Vaiani M, Cascella T, Bhoori S, Morosi C, et al. Development of a prognostic score to predict response to Yttrium-90 radioembolization for hepatocellular carcinoma with portal vein invasion. J Hepatol. 2018 Apr;68(4):724-32. 
34 Mohamad B, Shah V, Onyshchenko M, Elshamy M, Aucejo F, Lopez R, et al. Characterization of hepatocellular carcinoma (HCC) in non-alcoholic fatty liver disease (NAFLD) patients without cirrhosis. Hepatol Int. 2016 Jul; 10(4):632-9.

35 Jun TW, Yeh ML, Yang JD, Chen VL, Nguyen P, Giama NH, et al. More advanced disease and worse survival in cryptogenic compared to viral hepatocellular carcinoma. Liver Int. 2018 May;38(5):895-902.

36 Weinmann A, Alt Y, Koch S, Nelles C, Düber C, Lang H, et al. Treatment and survival of non-alcoholic steatohepatitis associated hepatocellular carcinoma. BMC Cancer. 2015 Apr;15(1):210.

37 Lee CH, Hsieh SY, Lin JL, Liu MS, Yen TH. Hepatocellular carcinoma in patients with chronic kidney disease. World J Gastroenterol. 2013 Apr;19(16):2466-72.

38 Kennoki T, Kondo T, Kimata N, Murakami J, Ishimori I, Nakazawa H, et al. Clinical results and pharmacokinetics of sorafenib in chronic hemodialysis patients with metastatic renal cell carcinoma in a single center. Jpn J Clin Oncol. 2011 May;41(5):647-55.

39 Garwood ER, Fidelman N, Hoch SE, Kerlan RK Jr, Yao FY. Morbidity and mortality following transarterial liver chemoembolization in patients with hepatocellular carcinoma and synthetic hepatic dysfunction. Liver Transpl. 2013 Feb;19(2):164-73.

40 Op den Winkel M, Nagel D, Op den Winkel P, Trojan J, Paprottka PM, Steib CJ, et al. Transarterial chemoembolization for hepatocellular carcinoma: development and external validation of the Munich-TACE score. Eur J Gastroenterol Hepatol. 2018 Jan;30(1):44-53. 